\title{
Immediate post placental insertion of IUD-the challenge to solve the expulsion problem
}

\section{Editorial}

Immediate postpartum intrauterine device (IUD) insertion deserves great attention as it can provide immediate contraception and prevents repeat unintended pregnancies. ${ }^{1}$ Immediate post placental insertion (within 10 minutes of delivery of the placenta) of copper-bearing or hormone-releasing IUDs is generally safe and acceptable, although compared with interval insertion it carries a higher risk of expulsion, thus affecting effectiveness and overall patient acceptance. ${ }^{2}$ Women undergoing Cesarean section need contraception, as an interdelivery interval shorter than 18 months is considered a risk factor for uterine rupture. ${ }^{3}$ In these women, a low expulsion risk is therefore paramount.

Over the past decades attempts have been made to solve the expulsion problem by modifying existing devices, such as adding absorbable sutures (Delta-T) or additional appendages. These attempts did not appear beneficial. Post placental placement of conventional IUDs during Cesarean section is associated with lower expulsion rates when compared to immediate postplacental vaginal insertion. Expulsion rates vary from around 5\% at 12 months to up to $50 \%$ and even higher if partial expulsions are included in the analysis. ${ }^{4-6}$

The risk of expulsion can be reduced substantially with appropriate training in postpartum insertion techniques. However, it appears that a simple and elegant solution to minimize the number of expulsions is to simply attach the IUD to the fundus of the uterus immediately postplacental.

An anchoring technique for use in the immediate postpartum period was developed in Belgium in the 1980s and has been the subject of extensive clinical research since 1985 at the University in Ghent and in internationally conducted clinical trials. ${ }^{7-9}$ Since its inception, the technology has passed through several phases of improvement. The last version was the subject of clinical trials conducted in China. The technique consisted in the suspension of a frameless, copper-releasing IUD immediately in the post placental uterus during Cesarean section. The anchor consisted of a biodegradable cone (polycaprolactone) which was added below the anchoring knot of the frameless IUD. The objective was to study the effects of immediate insertion of a frameless IUD during Cesarean section on the bleeding pattern, duration of lochia and healing of uterus. Two hundred women used the IUD and 204 women who did not use the IUD served as the control group. Followup visit was performed at 42 days and 90 days after delivery. There was no significant difference in postpartum hemorrhage, continuance of lochia, and healing of uterus was normal. The expulsion rate was $4 \% .{ }^{10}$ However, it was found that removal of the IUD was difficult in a significant number of women due to the slow degradation time of the cone material. It was concluded that further research on the improvement of absorption time of the biodegradable component may provide additional benefits of the retention technology.

Recently, the technique of suspending the frameless IUD for intracesarean insertion was further optimized. The technique consists of the precise placement of the anchoring knot immediately below the serosa of the uterus, followed by fixing the knot in place with an absorbable suture. The IUD tail is looped in the cervical canal and is cut prior to discharge from the hospital. In case the tail is in the
Volume 2 Issue 5 - 2015

\author{
Dirk A Wildemeersch,' 'Norman D \\ Goldstuck, ${ }^{2}$ Dirk RG Janssens ${ }^{3}$ \\ 'Gynecological Outpatient Clinic and IUD Training Center, \\ Belgium \\ ${ }^{2}$ Department of Obstetrics and Gynaecology, Stellenbosch \\ University, South Africa \\ ${ }^{3}$ Gynecological outpatient clinic, Belgium
}

Correspondence: Dirk A Wildemeersch, Gynecological Outpatient Clinic and IUD Training Center, Ghent, Belgium, Email d.wildemeersch@skynet.be

Received: July 21, 2015 | Published: July 30, 2015

cavity, it usually can be picked-up using a thin, $3 \mathrm{~mm}$ alligator forceps when removal is requested. The anchoring technique has shown to be easy, quick and safe in a pilot trial with no expulsions at 12 months. It was readily apparent that the technique could be considered a major advance, suitable for general use due to its simplicity requiring limited training. The position of the anchor in the fundus of the uterus can be identified using sonography by localizing the stainless steel marker attached to the anchoring knot. Although no removal studies have been conducted, removal of the IUD is expected to be similar to the removal after interval insertion of the device. ${ }^{11}$ A modified technique is currently been developed for insertion after vaginal delivery. We prefer the frameless IUD over framed IUDs as the latter may cause discrepancy with the uterine cavity and embedment during involution of the uterus, particularly during prolonged lactation as hyper involution in these women is not uncommon. ${ }^{12}$ Uterine compatibility will dictate patient continuation rates and overall patient acceptance. In addition, the availability of adequate contraception immediately post Cesarean delivery may have an added benefit in reducing the number of Cesarean sections performed worldwide. By allowing for adequate timing between pregnancies full uterine recover would be achieved thus allowing women to achieve vaginal delivery. Studies have shown that 40 to $80 \%$ of women can successful achieve vaginal births after Cesarean section (VBAC). ${ }^{13}$

Further studies should be initiated related to new suspension techniques as they appear to be the only solution to solve the expulsion problem associated with postpartum insertion of IUDs. They will also expand the method as a strategy to reduce unintended pregnancy and rapid repeat pregnancy in adolescents..$^{14}$

\section{Acknowledgments}

None.

\section{Conflicts of interest}

The authors declare there is no conflict of interests.

\section{References}

1. Rodriguez MI, Even M, Espey E. Advocating for immediate postpartum LARC: increasing access, improving outcomes, and decreasing cost. Contraception. 2014;90(5):468-471. 
2. American College of Obstetricians and Gynecologists. ACOG Practice Bulletin No. 121: long-acting reversible contraception: implants and intrauterine devices. Obstet Gynecol. 2011;118(1):184-196.

3. Bujold E, Gauthier RJ. Risk of Uterine Rupture Associated with an Interdelivery Interval Between 18 and 24 Months. Obstet Gynecol. 2010;115(5):1003-1006.

4. Kapp N, Curtis KM. Intrauterine device insertion during the postpartum period: a systematic review. Contraception. 2009;80(4):327-336.

5. Chi IC, Wilkens LR, Rogers S. Expulsions in immediate postpartum insertions of Lippes Loop D and Copper T IUDs and their counterpart Delta devices -- an epidemiological analysis. Contraception. 1385;32(2):119 134 .

6. Goldstuck ND, Steyn PS. Intrauterine contraception after cesarean section and during lactation: a systematic review. Int $J$ Womens Health. 2013;5:811-818.

7. Thiery M, Van Kets H, Van der Pas H. Immediate postplacental IUD insertion: The expulsion problem. Contraception. 1985;31(4):331-349.

8. Van Kets H, Parewijck W, Kleinhout J, et al. Clinical experience with the Gyne-T postpartum intrauterine device. Fertil Steril. 1991;55(6):11441149 .
9. Van Kets H, Parewijck W, Van der Pas H, et al. Immediate postplacental insertion and fixation of the CuFix postpartum implant system. Contraception. 1993;48(4):349-357.

10. Zhang H, Fang G, Zhou C. Study on GyneFix PP IUD insertion during cesarean section. Chinese Journal of Family Planning. 2004;12(8):481482 .

11. Batar I, Wildemeersch D. The force required to remove an anchored bioactive substance from the human uterus: results after long-term use. Contraception. 2004;69(6):501-503.

12. El-Minawi MF, Foda MS. Postpartum lactation amenorrhea: endometrial pattern and reproductive ability. Am J Obstet Gynecol. 1971;111(1):17-21.

13. Stamilio DM, DeFranco E, Paré E, et al. Short Interpregnancy Interval: Risk of Uterine Rupture and Complications of Vaginal Birth after Caesarean Delivery. Obstet Gynaecol. 2007;110(5):1075-1082.

14. Tocce KM, Sheeder JL, Teal SB. Rapid repeat pregnancy in adolescents: do immediate postpartum contraceptive implants make a difference? Am J Obstet Gynecol. 2012;206(6):481e1-481e7. 